\title{
MÉTODOS ESTOCÁSTICOS PARA MODELAGEM DE ESCOAMENTO ESTACIONÁRIO E TRANSIENTE EM MEIOS POROSOS
}

\author{
Juarez dos Santos Azevedo ${ }^{1,3}$, Saulo Pomponet Oliveira² e Olivar Antônio Lima de Lima ${ }^{3}$ \\ Recebido em 29 dezembro, 2008 / Aceito em 14 setembro, 2009 \\ Received on December 29, 2008 / Accepted on September 14, 2009
}

\begin{abstract}
One way to incorporate the uncertainty of field data and the spatial variability in the hydraulic properties of aquifers is to prescribe probability distributions to the material properties of the medium. The statistical properties of the hydraulic potential are then determined by the numerical solution of stochastic differential equations governing the flow in the aquifer. For this purpose we consider two stochastic finite element methods, Monte Carlo and stochastic Collocation, to estimate the mean and variance of the potential for saturated flow in statistically heterogeneous media, assuming that the coefficient of hydraulic transmissibility follows a lognormal distribution. One of the crucial issues in the accuracy of numerical methods is the spatial correlation length associated with the transmissibility. We also discuss some configurations of plant extraction that were recently proposed for a rational exploration of the Recôncavo aquifer in the Capivara river basin (Bahia, Brazil), by comparing two classical models and by introducing the randomness of transmissibility in one of these configurations.
\end{abstract}

Keywords: Karhunen-Loève expansion, stochastic finite element methods, free aquifer.

RESUMO. Uma maneira de incorporar a incerteza das medidas de campo e a variabilidade espacial nas propriedades hidráulicas de aqüíferos é estabelecer distribuições de probabilidade para os parâmetros físicos do meio. As propriedades estatísticas do potencial hidráulico são então determinadas pela solução numérica das equações diferenciais estocásticas que regem o regime de escoamento no aqüífero. Neste trabalho descrevemos a utilização de dois métodos de elementos finitos estocásticos (0 método de Monte Carlo e o método da Colocação) para estimar a média e a variância do potencial hidráulico para fluxo saturado em meio estatisticamente heterogêneo, supondo que o coeficiente de transmissividade hidráulica é descrito por um processo lognormal. Um dos fatores decisivos na precisão numérica dos métodos é 0 comprimento de correlação associado à transmissividade. Discutimos também algumas configurações de baterias de extração que foram recentemente propostas para uma exploração adequada do aqǘfero Recôncavo na bacia do rio Capivara (Bahia, Brasil), comparando dois modelos clássicos e introduzindo a aleatoriedade da transmissividade em um dos arranjos de poços que foram propostos.

Palavras-chave: expansão de Karhunen-Loève, métodos de elementos finitos estocásticos, aqüíferos livres.

\footnotetext{
1Laboratório Nacional de Computação Científica, Av. Getúlio Vargas, 333, 25651-075 Petrópolis, RJ, Brasil - E-mail: juarezsa@gmail.com

2DMAT-UFPR, Centro Politécnico, 81531-990 Curitiba, PR, Brasil - E-mail: saulopo@ufpr.br

${ }^{3}$ CPGG-UFBA, Instituto de Geociências, Campus Universitário de Ondina, 40170-290 Salvador, BA, Brasil - E-mail: olivar@cpgg.ufba.br
} 


\section{INTRODUÇÃo}

0 teste de bombeamento de poços é a técnica mais confiável para obter as características hidráulicas de sistemas aqüíferos. 0 exame detalhado das informações registradas, principalmente 0 rebaixamento induzido pelo bombeamento, requer 0 uso de modelos analíticos ou numéricos de escoamento para o poço. Os modelos analíticos são geralmente utilizados na análise de testes de bombeamento através de equações fluxo-poço sob hipóteses simplificadoras. Quando se usam modelos numéricos, as hipóteses podem ser menos restritivas, porém são introduzidos erros associados com as técnicas numéricas utilizadas.

Neste trabalho utilizamos métodos numéricos estocásticos para estimar a média e a variância da carga ou potencial hidráulico em modelos de escoamento em que a transmissividade hidráulica (ou condutividade hidráulica no regime permanente) segue uma distribuição de probabilidade lognormal. 0 conhecimento destas propriedades estatísticas é importante pois permite estabelecer um intervalo de confiança para solução numérica.

A fórmula de Theis foi empregada no estudo de escoamento transiente para poços como um resultado do bombeamento sob condições de fronteira horizontais que se estendem para 0 infinito. Critérios de otimização relacionados à geometria de localização dos poços são aplicados no desenvolvimento destas soluções. A fim de testar a validade da solução e prover informações adicionais sobre os testes de bombeamento fora do domínio da solução analítica, são utilizadas simulações numéricas de Monte Carlo e do método da Colocação. Uma compreensão quantitativa destes resultados poderá prover um guia na análise de rebaixamento do nível estático do poço em aqüíferos livres.

Na próxima seção introduzimos o modelo de escoamento transiente e revisamos algumas soluções clássicas associadas a meios homogêneos e ilimitados. Em seguida, descrevemos a discretização estocástica dos modelos pelos métodos de Monte Carlo e da Colocação. Apresentamos experimentos numéricos do modelo transiente considerado, mas também uma versão do modelo em regime permanente. Para o regime permanente, consideramos o problema clássico de cinco poços (five spot, Correa \& Loula (2008)), enquanto no regime transiente, adotamos o modelo utilizado por Cavalcanti (2006) para estudar o aqǘfero Recôncavo (BA).

\section{FORMULAÇ̃̃O DO PROBLEMA}

Considere um poço em um aqüífero livre cuja descarga é dada por uma taxa constante $Q$. A equação de continuidade e lei de
Darcy são descritas respectivamente por

$$
S(\mathbf{x}) \frac{\partial h(\mathbf{x} ; \boldsymbol{\omega} ; t)}{\partial t}+\nabla \cdot \mathbf{q}(\mathbf{x} ; \boldsymbol{\omega} ; t)=f(\mathbf{x})
$$

e

$$
\mathbf{q}(\mathbf{x} ; \boldsymbol{\omega} ; t)=-T(\mathbf{x} ; \omega) \nabla h(\mathbf{x} ; \omega ; t),
$$

em que $\mathbf{q}(\mathbf{x} ; \boldsymbol{\omega} ; t)$ é o vetor de descarga específica, $S(\mathbf{x}) 0$ coeficiente de armazenamento elástico, $T(\mathbf{x} ; \boldsymbol{\omega}) 0$ campo de transmissividade hidráulica e $f(\mathbf{x})=Q \cdot \delta(\mathbf{x})$, sendo $\delta(\mathbf{x})$ a função delta de Dirac. Determinamos a carga hidráulica $h(\mathbf{x} ; \boldsymbol{\omega} ; t)$ por meio das equações (1)-(2) em conjunto com as condições iniciais e de fronteira

$$
\begin{cases}h(\mathbf{x} ; \boldsymbol{\omega} ; 0)=H_{0}, & \mathbf{x} \in D \subset \mathbb{R}^{2} \\ h(\mathbf{x} ; \boldsymbol{\omega} ; t)=h_{0}(r) & \mathbf{x} \in \Gamma_{D},\end{cases}
$$

que estabelecem que a carga hidráulica é constante $\left(H_{0}\right)$ até 0 início do bombeamento do poço e que é dada pela carga prescrita $h_{0}(r)$ na fronteira $\Gamma_{D}$ formada por pontos a uma distância radial $r$ do poço suficientemente grande. Esta condição visa aproximar

$$
\lim _{\|\mathbf{x}\| \rightarrow \infty} h(\mathbf{x} ; \boldsymbol{\omega} ; t)=H_{0} .
$$

Em particular, utilizamos as funções $h_{0}(r)$ calculadas a partir das soluções determinísticas $h=h(\mathbf{x} ; t)$ dadas pelas fórmulas de Boulton (6) e Theis (8). Podemos estender o modelo para um arranjo de poços modificando a função $f(\mathbf{x})$.

Introduzimos a incerteza do modelo, quantificada pela variável independente $\boldsymbol{\omega}$, por meio da transmissividade $T(\mathbf{x} ; \boldsymbol{\omega})$. A transmissividade forma uma função aleatória de representação lognormal, ou seja:

$$
T(\mathbf{x} ; \boldsymbol{\omega})=\exp (Y(\mathbf{x} ; \boldsymbol{\omega}))
$$

sendo $Y(\mathbf{x} ; \boldsymbol{\omega})$ um processo Gaussiano e estatisticamente estacionário. Admitimos também que a função de covariância $C(\mathbf{x}, \mathbf{y})$ do processo $Y(\mathbf{x} ; \boldsymbol{\omega})$ satisfaz $C(\mathbf{x}, \mathbf{y})=C(\mathbf{y}, \mathbf{x})$ e $C(\mathbf{x}, \mathbf{x})>0$ para todo $\mathbf{x} \neq \mathbf{0}$. Deste modo, $Y(\mathbf{x} ; \boldsymbol{\omega})$ pode ser representado em termos da expansão de Karhunen-Loève

$$
Y(\mathbf{x} ; \boldsymbol{\omega})=\langle Y(\mathbf{x} ; \boldsymbol{\omega})\rangle+\sum_{n=1}^{\infty} \sqrt{\lambda_{n}} \varphi_{n}(\mathbf{x}) \xi_{n},
$$

em que $\langle Y(\mathbf{x} ; \boldsymbol{\omega})\rangle$ é a média de $Y$ e os termos $\lambda_{n}$ e $\varphi_{n}(\mathbf{x})$ representam 0 autovalor e a autofunção respectivamente, associados a $C(\mathbf{x}, \mathbf{y})$. Por outro lado, $\left\{\xi_{1}, \xi_{2}, \ldots\right\}$ é um conjunto de variáveis aleatórias Gaussianas mutuamente ortonormais com média zero. 
Para fins de cálculo, é necessário truncar a expansão acima, reduzindo a transmissividade à forma $T(\mathbf{x} ; \boldsymbol{\omega}) \approx$ $T\left(\mathbf{x} ; \xi_{1}, \ldots, \xi_{M}\right)$. 0 termo $M$ é 0 número de coordenadas efetivas da caracterização estocástica e será referido como a dimensão estocástica.

\section{Fórmula de Boulton}

A fórmula de Boulton supõe que o bombeamento produz uma resposta não instantânea do aqüífero e pode ser utilizada para aproximar o fluxo não estacionário em um sistema aqǘf́ero livre que possui um aqüiclude na base e uma superfície livre em seu topo, com fluxo vertical entre as fronteiras (Hunt \& Scott, 2005).

Boulton (vide Hantush (1964)) verificou que o rebaixamento induzido $s=s(\mathbf{x} ; t)$ em um poço num aqüífero homogêneo cujo raio de influência é infinito e sem fonte de recarga pode ser expresso pela fórmula

$$
s=H_{0}-h=\frac{Q}{2 \pi T} V\left(\frac{K t}{\epsilon H_{0}}, \frac{v}{H_{0}}\right),
$$

cuja função $V(\tau, \rho)$, chamada de função de poço por gravidade, é definida pela integral

$$
\begin{gathered}
V(\tau, \rho)=\int_{0}^{\infty} \frac{1}{u} J_{0}(u, \rho) \\
\times[1-\exp (-\tau \cdot u \cdot \tanh (u))] d u,
\end{gathered}
$$

com

$$
\tau=K t / \epsilon H_{0}, \quad \rho=v / H_{0}, \quad v=T / S \approx \epsilon
$$

e $J_{0}$ é a função de Bessel de primeira espécie e ordem zero. A transmissividade $T$ e 0 coeficiente de armazenamento específico $S$ são constantes. Em particular, no caso de aqǘf́eros com espessura uniforme, o coeficiente de transmissividade é fixado em $T=H_{0} K$ onde $K$ é a condutividade hidráulica. 0 parâmetro $\epsilon$ é 0 suprimento específico do aquífero ou a razão entre o volume da água que uma rocha ou solo produz por gravidade e o seu próprio volume, sendo aproximada pela porosidade efetiva $\psi_{e}$ (porção do espaço poroso conectado no material permeável e saturado no qual o fluxo ocorre).

\section{Fórmula de Theis}

Sejam $h_{w}$ a altura de água no poço e $r_{w} 0$ raio do poço de bombeamento. Hantush (1964) notou que quando $t>30 r_{w}^{2} / v$ e a profundidade de saturação na região onde 0 fluxo não perturbado é menor que a altura da coluna de água no poço em bombeamento $\left(0.5 H_{0}<h_{w}\right)$, o rebaixamento observado é dado pela expressão

$$
s=H_{0}^{2}-h^{2}=\frac{Q}{2 \pi K} W\left(\frac{r^{2}}{4 v t}\right),
$$

com $W(u)$ sendo a função de poço para aqüíferos sem vazamento definida por

$$
W(u)=\int_{u}^{\infty} \frac{1}{x} \exp (-x) d x .
$$

A Eq. (8) é conhecida como a solução de Theis para aqüíferos livres e foi utilizada por Cavalcanti (2006) para 0 cálculo do rebaixamento induzido por baterias de poços de produção e para a modelagem analítica da interferência entre poços e da proximidade destas como fronteiras verticais. Porém, a fórmula de Theis é muito restritiva visto que supõe que o fluxo é horizontal, ao contrário da fórmula de Boulton que faz uma extensão tanto para a velocidade horizontal quanto a vertical do fluxo (Hunt \& Scott, 2005).

\section{Descarga de poços interferentes}

De acordo com Hantush (1964), se a localização de $N$ poços e o nível de água em cada um deles ao final de um dado período de bombeamento contínuo forem conhecidos, então a descarga de cada poço pode ser obtida resolvendo as $N$ equações linearmente independentes, para um dado nível de água em cada poço usando a equação

$$
s=\sum_{i=1}^{N} Q_{i} Z_{w i}\left(\mathbf{x}, t_{i}\right)
$$

em que $Q_{i} Z_{w i}$ é o potencial do fluxo freático no $i$-ésimo poço, ou seja, o lado direito de uma equação de rebaixamento de um particular sistema de fluxo (a solução de Boulton (6) ou a solução de Theis (8)) e $Q_{i}$ representa a descarga (que pode ser negativa ou positiva).

Pela solução de Theis (8), dois poços freáticos de raio $r_{w}$ afastados lateralmente por uma distância $d_{m}$, bombeados pelo mesmo período de tempo $t_{0}$ num aqǘf́ero sem vazamento e rebaixamento $s$ para ambos os poços, terão descargas $Q_{1}$ e $Q_{2}$ dadas por

$$
Q_{1}=Q_{2}=\frac{2 \pi K s}{W\left(\frac{r_{w}^{2}}{4 v t_{0}}\right)+W\left(\frac{d_{m}^{2}}{4 v t_{0}}\right)} .
$$

Similarmente para três poços distribuídos na forma de um triângulo equilátero de lado $d_{m}$,

$$
Q_{1}=Q_{2}=Q_{3}=\frac{2 \pi K s}{W\left(\frac{r_{w}^{2}}{4 v t_{0}}\right)+2 W\left(\frac{d_{m}^{2}}{4 v t_{0}}\right)} .
$$


Quando são feitas estimativas de rebaixamentos próximos a poços de bombeamento, é necessário incluir a influência de outros poços operando na área, pois a descarga $Q$ e 0 rebaixamento $s$ são individualmente afetados por poços vizinhos, exceto nos casos em que o período de bombeamento é muito curto ou 0 espaçamento entre os poços seja grande o bastante para que as zonas de influência não se sobreponham efetivamente.

\section{METODOLOGIA}

Para estudar o efeito da incerteza na transmissividade $T(\mathbf{x} ; \boldsymbol{\omega})$ sobre a carga hidráulica $h(\mathbf{x} ; \boldsymbol{\omega} ; t)$, calculamos a média e a variância da solução do problema definida pelas equações (1)(3). Para aproximar esta solução, discretizamos as variáveis espaciais, temporais e estocásticas separadamente. A discretização espacial é feita pelo método de elementos finitos, enquanto a discretização temporal é realizada pelo método de CrankNicolson. Para a discretização estocástica do problema (1)-(3), consideramos os métodos de Monte Carlo e da Colocação, descritos a seguir.

\section{Método de Monte Carlo (MC)}

0 método de Monte Carlo depende da geração de um grande número de amostras do campo de transmissividade hidráulica (4). Para calcular este campo primeiro discretizamos o domínio espacial $D$ em uma malha formada por $N$ vértices. Em seguida, avaliamos a função de covariância $C(\mathbf{x}, \mathbf{y})$ nos vértices da maIha, gerando uma matriz simétrica e positiva definida $\mathbf{C}$ de tamanho $N \times N$ dada por $C_{i, j}=C\left(\mathbf{x}_{i}, \mathbf{y}_{j}\right)(1 \leq i, j \leq N)$. Valendo-se da decomposição de Cholesky, esta matriz pode ser decomposta na forma $\mathbf{C}=\mathbf{L L}^{T}$, sendo que $\mathbf{L}$ é uma matriz triangular inferior.

Seguindo Chakraborty \& Dey (1995), aproximamos $Y(\mathbf{x} ; \boldsymbol{\omega})$ nos vértices da malha pelo vetor aleatório $\mathbf{Y}=\mathbf{Y}(\boldsymbol{\omega})$ dado por $\mathbf{Y}=\mathbf{Y}_{0}+\mathbf{L} \boldsymbol{\xi}$, onde $\mathbf{Y}_{0}$ corresponde à média de $Y$ avaliada nos vértices da malha e $\boldsymbol{\xi}=\left(\xi_{1}, \ldots, \xi_{N}\right)^{T}$ é um vetor de $N$ variáveis aleatórias independentes e identicamente distribuídas com média zero e variância unitária. Note que a covariância do vetor aleatório $\mathbf{Y}$ coincide com a matriz $\mathbf{C}$ :

$$
\left\langle\left(\mathbf{Y}-\mathbf{Y}_{0}\right)\left(\mathbf{Y}-\mathbf{Y}_{0}\right)^{T}\right\rangle=\mathbf{L}\left\langle\boldsymbol{\xi} \xi^{T}\right\rangle \mathbf{L}^{T}=\mathbf{L} \mathbf{I} \mathbf{L}^{T}=\mathbf{C} .
$$

A metodologia prossegue com a geração das amostras $\boldsymbol{\xi}^{(1)}, \boldsymbol{\xi}^{(2)}, \ldots, \boldsymbol{\xi}^{\left(N_{A}\right)}$ do vetor $\boldsymbol{\xi}=\boldsymbol{\xi}(\boldsymbol{\omega})$. Para cada $k=$ $1,2, \ldots, N_{A}$, seja $T^{(k)}(\mathbf{x})=T\left(\mathbf{x} ; \boldsymbol{\xi}^{(k)}\right)$ a função que interpola $\exp \left(\mathbf{Y}^{(k)}\right)=\exp \left(\mathbf{Y}_{0}+\mathbf{L} \boldsymbol{\xi}^{(k)}\right)$ nos vértices da maIha, isto é, $T^{(k)}\left(\mathbf{x}_{i}\right)=\exp \left(Y_{i}^{(k)}\right)$. Calculamos para cada $k$ a solução $h^{(k)}(\mathbf{x} ; t)$ do problema

$$
\left\{\begin{array}{l}
S(\mathbf{x}) \frac{\partial h^{(k)}(\mathbf{x} ; t)}{\partial t}-\nabla \\
\quad \times\left(T^{(k)}(\mathbf{x}) \nabla h^{(k)}(\mathbf{x} ; t)\right)=f(\mathbf{x}) \\
h^{(k)}(\mathbf{x} ; 0)=H_{0}, \quad \mathbf{x} \in D \subset \mathbb{R}^{2} \\
h^{(k)}(\mathbf{x} ; t)=h_{0}(r) \quad r \in \Gamma_{D},
\end{array}\right.
$$

que aproximamos pelo método de elementos finitos no espaço e o método de Crank-Nicolson no tempo. Convém utilizar no método de elementos finitos a mesma malha e as mesmas funções de interpolação usadas no procedimento acima descrito.

As soluções $h^{(1)}(\mathbf{x} ; t), h^{(2)}(\mathbf{x} ; t), \ldots, h^{\left(N_{A}\right)}(\mathbf{x} ; t)$ do problema (13) representam amostras que podemos utilizar para estimar a média e a variância da solução procurada. Em geral, um alto número de amostras fornece uma aproximação precisa da média e da variância, mas eleva o custo computacional.

\section{Método da Colocação (MCol)}

Enquanto o método de Monte Carlo introduz uma aproximação da transmissividade diretamente nas equações diferenciais (1)-(2), 0 método da Colocação parte do problema variacional associado

$$
\begin{gathered}
\int_{D}\left(\frac{\partial h(\mathbf{x} ; \boldsymbol{\omega}, t)}{\partial t} \phi(\mathbf{x})+T(\mathbf{x} ; \boldsymbol{\omega}) \nabla h(\mathbf{x} ; \boldsymbol{\omega} ; t) \nabla \phi(\mathbf{x})\right) d \mathbf{x} \\
=\int_{D} f(\mathbf{x}) \phi(\mathbf{x}) d \mathbf{x}, \quad \forall \phi \in H_{0}^{1}(D),
\end{gathered}
$$

onde $H_{0}^{1}(D)$ é o espaço de funções de quadrado integrável cuja derivada é quadrado integrável e com suporte compacto em $D$ (Evans, 1998). Em seguida, aproximamos $T(\mathbf{x} ; \boldsymbol{\omega})$ e $h(\mathbf{x} ; \boldsymbol{\omega} ; t)$ pelas expansões finitas

$$
\begin{gathered}
T(\mathbf{x} ; \boldsymbol{\omega}) \approx \exp \left(\langle Y(\mathbf{x}, \boldsymbol{\omega})\rangle+\sum_{n=1}^{M} \sqrt{\lambda_{n}} \varphi_{n}(\mathbf{x}) \xi_{n}\right), \\
h(\mathbf{x} ; \boldsymbol{\omega} ; t) \approx \sum_{k=1}^{M_{p}} h^{(k)}(\mathbf{x}, t) L_{k}(\boldsymbol{\xi})
\end{gathered}
$$

onde as funções $L_{k}(\boldsymbol{\xi})$ satisfazem $L_{k}\left(\boldsymbol{\xi}^{(l)}\right)=\delta_{k, l}$. Substituindo (15) em (14) e avaliando a equação resultante em $\boldsymbol{\xi}=$ $\boldsymbol{\xi}^{(1)}, \boldsymbol{\xi}^{(2)}, \ldots, \boldsymbol{\xi}^{\left(M_{p}\right)}$, obtemos

$$
\begin{gathered}
\int_{D}\left(\frac{\partial h^{(k)}(\mathbf{x}, t)}{\partial t} \phi(\mathbf{x})+T\left(\mathbf{x}, \boldsymbol{\xi}^{(k)}\right) \nabla h^{(k)}(\mathbf{x}, t) \nabla \phi(\mathbf{x})\right) d \mathbf{x} \\
=\int_{D} f(\mathbf{x}) \phi(\mathbf{x}) d \mathbf{x} \quad \forall \phi \in H_{0}^{1}(D),
\end{gathered}
$$

para $k=1,2, \ldots M_{p}$, de modo análogo ao método de Monte Carlo.

Observe que a expansão de $T(\mathbf{x} ; \boldsymbol{\omega})$ em (15) decorre do truncamento da expansão de Karhunen-Loève (5). A aproximação 
estabelecida para $h(\mathbf{x} ; \boldsymbol{\omega} ; t)$ visa projetar a solução no espaço $H_{0}^{1}(D) \otimes \mathcal{P}_{p}(\Omega)$ em que $\mathcal{P}_{p}(\Omega) \subset L^{2}(\Omega)$ é gerado pelo produto tensorial de espaços de polinômios na forma

$$
\begin{aligned}
\mathcal{P}_{p}(\Omega)= & \bigotimes_{n=1}^{M} \mathcal{P}_{p_{n}}\left(\Omega_{n}\right), \\
\mathcal{P}_{p_{n}}\left(\Omega_{n}\right)= & \left\{a_{0}+a_{1} \xi_{n}+a_{2} \xi_{n}^{2}+\ldots+a_{p_{n}} \xi_{n}^{p_{n}},\right. \\
& \left.a_{0}, \ldots, a_{m} \in \mathbb{R}\right\} .
\end{aligned}
$$

Note que cada espaço $\mathcal{P}_{p_{n}}\left(\Omega_{n}\right)$ é formado por polinômios $p\left(\xi_{n}\right)$ de grau $p_{n}$ que dependem da variável aleatória $\xi_{n}$. Assim, a dimensão de $\mathcal{P}_{p_{n}}\left(\Omega_{n}\right)$ é $p_{n}+1$ e a dimensão de $\mathcal{P}_{p}$, que corresponde ao número de termos da expansão de $h(\mathbf{x} ; \boldsymbol{\omega} ; t)$, é dada por

$$
M_{p}=\prod_{n=1}^{M}\left(p_{n}+1\right) .
$$

Por exemplo, se $p_{1}=\ldots=p_{n}=P$, então $M_{p}=$ $(P+1)^{M}$.

0 conjunto $\mathcal{P}_{p}(\Omega)$ é um exemplo de espaço de polinômios de caos de dimensão finita (Rupert \& Miller, 2007), e é tradicionalmente escrito em termos de polinômios de Hermite, que são ortogonais com respeito à medida de probabilidade Gaussiana. Devido à ortogonalidade, as raízes destes polinômios definem fórmulas de quadratura Gaussiana. Em particular, as raízes

$$
\xi_{n}^{\left(k_{n}\right)}, k_{n}=1, \ldots, p_{n}+1
$$

do polinômio de Hermite $H_{n+1}\left(\xi_{n}\right)$ de grau $p_{n}+1$ satisfazem

$$
\begin{aligned}
\langle f\rangle & =\int_{\Omega_{n}} f(\xi) d \xi \\
& =\sum_{k_{n}=1}^{p_{n}+1} w_{k_{n}} f\left(\xi_{n}^{\left(k_{n}\right)}\right) \quad \forall f \in \mathcal{P}_{2 p_{n}+1},
\end{aligned}
$$

onde $w_{k_{n}}=(n+1) ! /\left(H_{n+2}\left(\xi_{n}^{\left(k_{n}\right)}\right)\right)^{2}$. Seguindo Babuška et al. (2007), definimos

$$
\begin{gathered}
\boldsymbol{\xi}^{(1)}, \boldsymbol{\xi}^{(2)}, \ldots, \boldsymbol{\xi}^{\left(M_{p}\right)}, \\
\boldsymbol{\xi}^{(k)}=\left(\xi_{1}^{\left(k_{1}\right)}, \xi_{2}^{\left(k_{2}\right)}, \ldots, \xi_{M}^{\left(k_{M}\right)}\right),
\end{gathered}
$$

e representamos a projeção de $h(\mathbf{x} ; \boldsymbol{\omega} ; t)$ através de $L_{k}(\boldsymbol{\xi})=$ $\prod_{n=1}^{M} L_{k_{n}}\left(\xi_{n}\right)$, onde $L_{k_{n}}\left(\xi_{n}\right) \in \mathcal{P}_{p_{n}}$ são polinômios interpoladores de Lagrange definidos por

$$
L_{k_{n}}\left(\xi_{n}^{\left(j_{n}\right)}\right)=\delta_{k_{n}, j_{n}} ; \quad j_{n}, k_{n}=1, \ldots, p_{n}+1 .
$$

Uma vez calculados $h^{(1)}(\mathbf{x}, t), \ldots, h^{\left(M_{p}\right)}(\mathbf{x}, t)$ que dependem de uma discretização no tempo e no espaço, como no método de Monte Carlo), a média aproximada é expressa por

$$
\begin{aligned}
E_{p}[h] & =\sum_{k=1}^{M_{p}} h^{(k)}(\mathbf{x}, t) E\left[L_{k}(\boldsymbol{\xi})\right] \\
& =\sum_{k=1}^{M_{p}} h^{(k)}(\mathbf{x}, t) w_{k}, \quad w_{k}=\prod_{n=1}^{M} w_{k_{n}} .
\end{aligned}
$$

De forma análoga, a variância aproximada é dada por

$$
\begin{gathered}
\operatorname{var}_{p}(h)=\sum_{i=1}^{M_{p}} \sum_{j=1}^{M_{p}}\left(h^{(i)}(\mathbf{x}, t)\right. \\
\left.-E_{p}[h]\right) w_{i} w_{j}\left(h^{(j)}(\mathbf{x}, t)-E_{p}[h]\right) .
\end{gathered}
$$

Note que a ordenação dos vetores $\boldsymbol{\xi}^{(k)}$, ou seja, a relação entre 0 índice $k$ e os índices $k_{1}, \ldots, k_{M}$ em (18), pode se tornar trabalhosa e 0 número total de vetores $M_{p}$ pode se tornar elevado à medida que $M$ e $p$ crescem. Esta característica do método da Colocação é conhecida como maldição da dimensionalidade (curse of dimensionality (Babuška et al., 2007)) e pode comprometer a eficiência do método.

\section{RESULTADOS}

Nesta seção descrevemos os resultados de dois experimentos que fornecem uma idéia geral da validade dos métodos numéricos acima descritos em problemas de escoamento laminar em aqüíferos. No primeiro experimento, consideramos o escoamento em regime permanente segundo o modelo clássico de five spot. No segundo experimento o escoamento étransiente e utiliza uma geometria motivada pelo estudo de otimização de Cavalcanti (2006). Além disso, o conjunto de parâmetros hidráulicos utilizados são compatíveis com os do aqüífero Recôncavo (BA).

Ambos os experimentos foram estudados no caso determinístico (Cavalcanti, 2006; Correa \& Loula, 2008). Propomos um estudo estocástico de tais problemas supondo que a transmissividade (ou condutividade hidráulica no regime permanente) segue uma distribuição lognormal com a função de covariância descrita a seguir.

\section{Função de covariância}

Vamos considerar o domínio espacial na forma $[0, L] \times[0, L]$ e uma função de covariância do processo $Y=Y(\mathbf{x} ; \boldsymbol{\omega})$ presente 
em (4) na forma

$$
\begin{aligned}
C(\mathbf{x}, \mathbf{y}) & =C\left(\left(x_{1}, x_{2}\right),\left(y_{1}, y_{2}\right)\right) \\
& =\sigma_{Y}^{2} \exp \left(-\left|x_{1}-y_{1}\right| / \eta-\left|x_{2}-y_{2}\right| / \eta\right)
\end{aligned}
$$

em que $\sigma_{Y}^{2}$ e $\eta$ são a variância e 0 comprimento de correlação do processo $Y$, respectivamente. No texto, o comprimento de correlação $\eta$ e 0 comprimento do intervalo $L$ possuem unidade de medida em metros. Neste caso, existem fórmulas explícitas (Zhang \& Lu, 2004) para os autovalores e as autofunções associadas a $C(\mathbf{x}, \mathbf{y})$, que são utilizadas na expansão de Karhunen-Loève (5):

$$
\begin{aligned}
& \lambda_{n}=\frac{4 \eta^{2} \sigma_{Y}^{2}}{\left(\eta^{2} \gamma_{i}^{2}+1\right)\left(\eta^{2} \gamma_{j}^{2}+1\right)}, \\
& \varphi_{n}(\mathbf{x})=\varphi_{i}\left(x_{1}\right) \varphi_{j}\left(x_{2}\right), \quad n=1,2, \ldots
\end{aligned}
$$

Nestas expressões o parâmetro $\gamma_{i}$ é encontrado a partir da equação transcendente

$$
\left(\eta^{2} \gamma^{2}-1\right) \sin (\gamma L)=2 \eta \gamma \cos (\gamma L),
$$

enquanto

$$
\begin{aligned}
\varphi_{k}(x)= & \left(\frac{\eta \gamma_{k}}{\sqrt{\left(\eta^{2} \gamma_{k}^{2}+1\right) L / 2+\eta}}\right) \cos \left(\gamma_{k} x\right) \\
& +\left(\frac{1}{\sqrt{\left(\eta^{2} \gamma_{k}^{2}+1\right) L / 2+\eta}}\right) \sin \left(\gamma_{k} x\right) .
\end{aligned}
$$

A relação entre o índice $n$ e os índices $\{i, j\}$ é escolhida de modo que os autovalores $\lambda_{n}$ estejam em ordem decrescente.

A aproximação de perturbação usada na derivação da média do potencial ficou restrita a uma pequena variabilidade do meio $\left(\sigma_{Y}^{2}=1\right)$. Todavia, podemos estender estes resultados para valores mais elevados de $\sigma_{Y}^{2}$.

Em ambos os experimentos vamos utilizar o método de elementos finitos com funções de base contínuas e bilineares por partes (Hughes, 1987) em uma malha uniforme de $20 \times 20$ elementos quadrados. Conduzimos simulações de Monte Carlo com 5000 amostras.

\section{Escoamento em regime permanente}

Consideramos o modelo de cinco poços (five spot) de escoamento em regime permanente gerado por um arranjo quadrangular de quatro poços de injeção nos vértices e com um poço de bombeamento no centro. Por simplicidade apenas um quarto do domínio é levado em conta e o fluxo prescrito na fronteira é zero. A injeção é efetuada no ponto $\mathbf{x}_{1}=(L, L), L=1,854075$, enquanto 0 bombeamento ocorre no ponto $\mathbf{x}_{2}=(0,0)$, com cargas $Q$ e $-Q$ respectivamente, com $Q=0,25 \mathrm{~m}^{3} / \mathrm{s}$ (vide Fig. 1(a)).

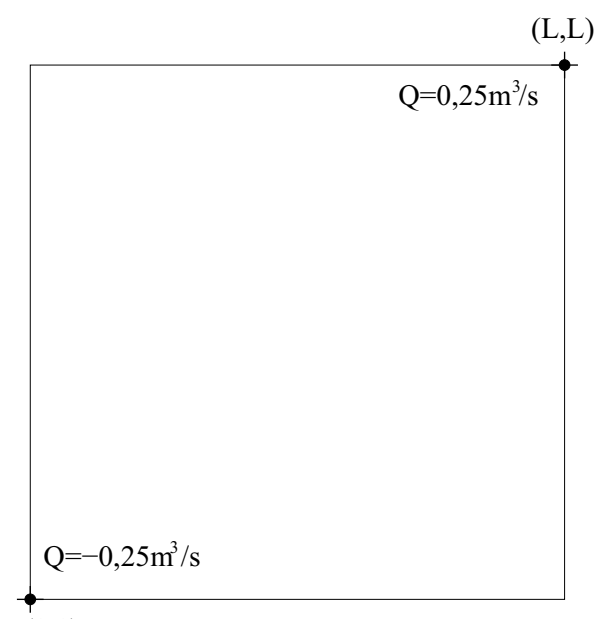

(a) $(0,0)$

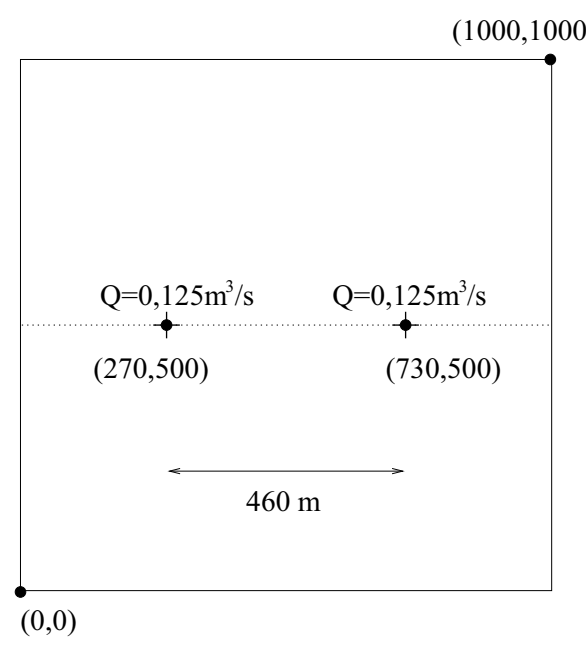

Figura 1 - Condições de Fronteira dos problemas de escoamento permanente (a) e escoamento transiente (b).

No regime permanente, as equações (1)-(2) se reduzem a

$$
\begin{gathered}
-\nabla \cdot(K(\mathbf{x} ; \boldsymbol{\omega}) \nabla h(\mathbf{x} ; \omega ; t))=f(\mathbf{x}), \\
f(\mathbf{x})=Q \cdot \delta\left(\mathbf{x}-\mathbf{x}_{1}\right)-Q \cdot \delta\left(\mathbf{x}-\mathbf{x}_{2}\right),
\end{gathered}
$$

em que $K(\mathbf{x} ; \boldsymbol{\omega})$ representa a condutividade hidráulica. Introduzimos a regularização da fonte $f(\mathbf{x})$ segundo Correa \& Loula (2008): as cargas nas coordenadas são aproximadas por cargas uniformemente distribuídas sobre os vértices dos elementos de modo que

$$
Q_{I}=\frac{0,25}{\left|D_{I}\right|}, \mathbf{x} \in D_{I} \quad Q_{B}=-\frac{0,25}{\left|D_{B}\right|}, \mathbf{x} \in D_{B},
$$


em que $\left|D_{I}\right|$ e $\left|D_{B}\right|$ são as áreas dos elementos contendo a injeção e 0 bombeamento, respectivamente.

As Figuras 2 e 3 apresentam os mapas iso-contorno e os perfis hidráulicos através dos poços na diagonal para a média e a variância do potencial respectivamente no caso em que as variáveis espaciais estão fracamente correlacionadas $(\eta=1)$. Verificamos que tanto a média do MCol quanto a variância possuem um pequeno desvio sobre os resultados do MC. Uma possível razão é 0 baixo coeficiente de correlação que tende a aumentar o desvio das variáveis espaciais na função de covariância. Aumentando-se 0 valor de $\eta$ para 4, os resultados da média e variância do MCol convergem para os resultados de MC demonstrando a precisão do modelo proposto (vide Figs. 4 e 5).

Notamos também um aumento da variância nas extremidades da fronteira em ambos os modelos MC e MCol. Como 0 fluxo próximo a fronteira é fixado, um pequeno valor relativo da condutividade hidráulica $K$ no elemento de fronteira sob uma realização, deverá forçar os pontos nodais na fronteira a terem um potencial elevado e conseqüentemente produzir um gradiente de potencial relativamente grande. Similarmente um grande valor de $K$ nos elementos de fronteira deverá forçar os nós da fronteira a terem pequeno valor do potencial, produzindo com isso um gradiente de potencial relativamente baixo. Desta maneira, a variação do potencial nos pontos nodais da fronteira deverá ser maior do que nos outros nós (Zhang \& Lu, 2002).

\section{Escoamento em regime transiente}

Para ilustrar a aproximação estocástica na determinação do cálculo de rebaixamento de poços e a validade desta aproximação, consideramos 0 problema descrito pela Figura $1(\mathbf{b})$. Utilizamos as equações (1)-(3), sendo $f(\mathbf{x})=Q \cdot \delta\left(\mathbf{x}-\mathbf{x}_{1}\right)-Q \cdot \delta(\mathbf{x}-$ $\mathbf{x}_{2}$ ). A taxa de bombeamento foi fixada em $Q=0,125 \mathrm{~m}^{3} / \mathrm{s}$ e os poços se localizam nas posições $\mathbf{x}_{1}=(270,500) \mathrm{e}$ $\mathbf{x}_{2}=(730,500)$. A transmissividade média é dada por $T_{G}=$ $\exp (\langle Y\rangle)=0,0038 \mathrm{~m}^{2} / \mathrm{s}$ e 0 tempo total de observação foi de um ano. (a)

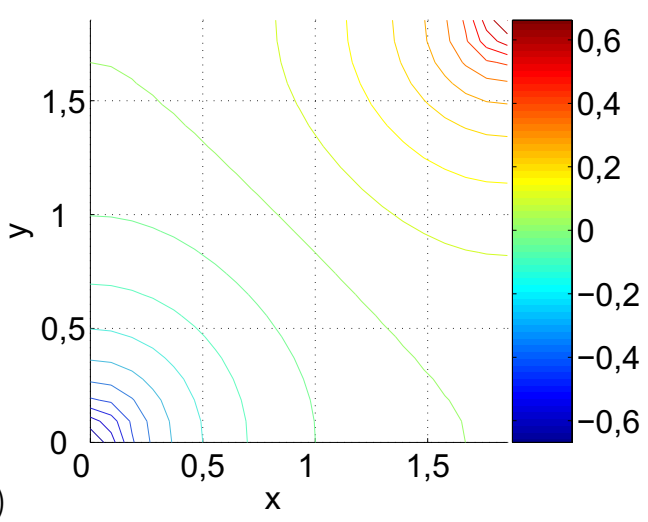

(b)

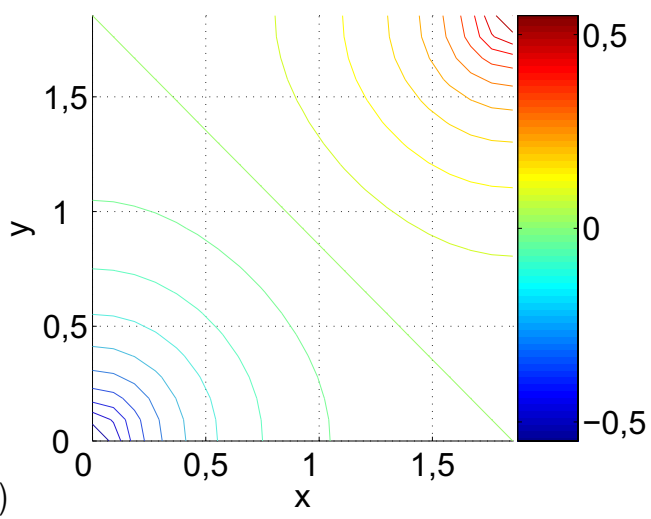

(c)

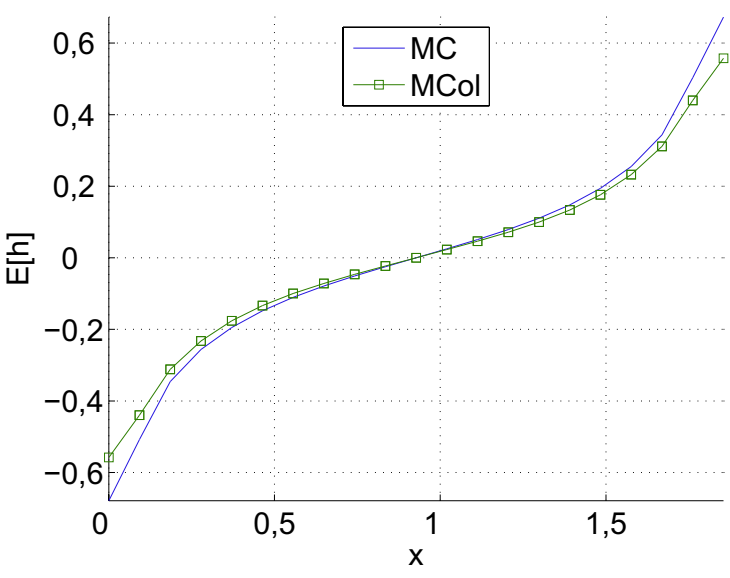

Figura 2 - Gráfico de Contorno da Média: (a) MC e (b) MCol. A Fig. (c) representa os perfis da diagonal com $\eta=1$ e $\sigma_{Y}^{2}=1$. 
(a)

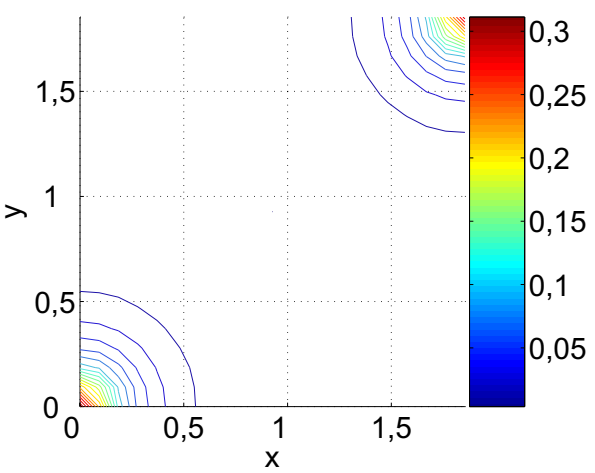

(c)

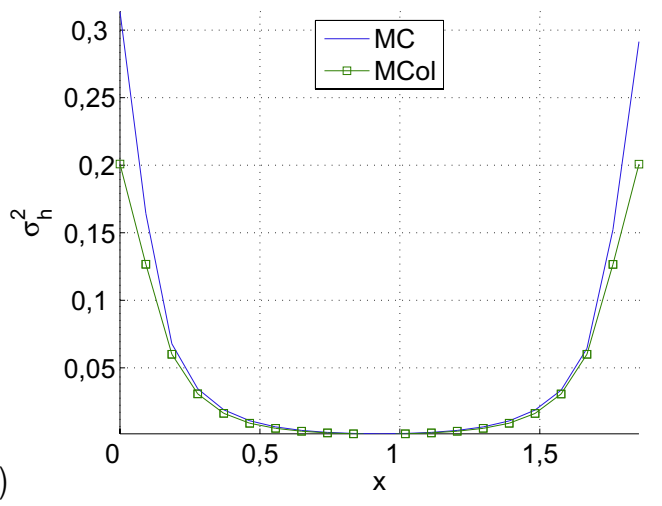

Figura 3 - Gráfico de Contorno da Variância: (a) MC e (b) MCol. A Fig. (c) representa os perfis da diagonal com $\eta=1$ e $\sigma_{Y}^{2}=1$.

(a)

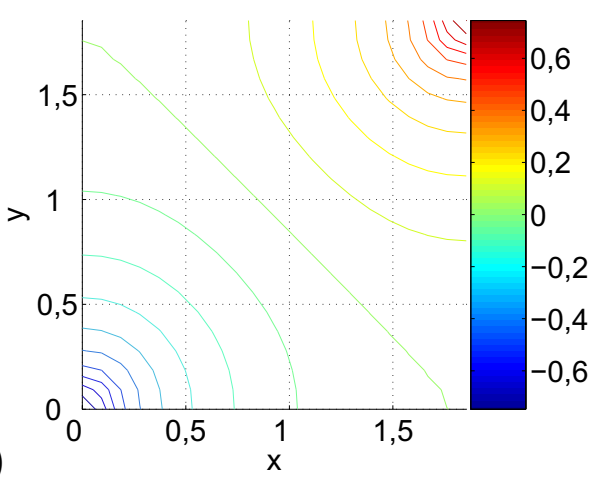

(b)

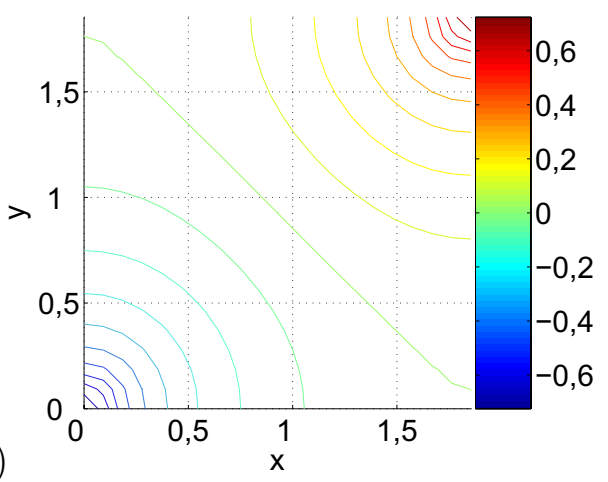

(c)

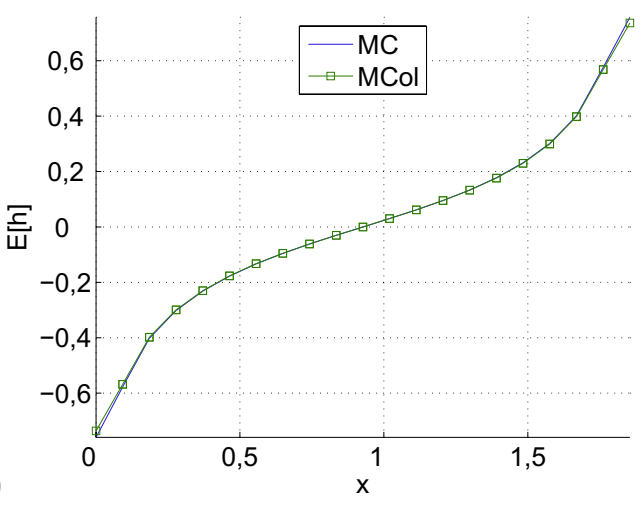

Figura 4 - Grático de Contorno da Média: (a) MC e (b) MCol. A Fig. (c) representa os perfis da diagonal com $\eta=4 \mathrm{e} \sigma_{Y}^{2}=1$. 
(a)

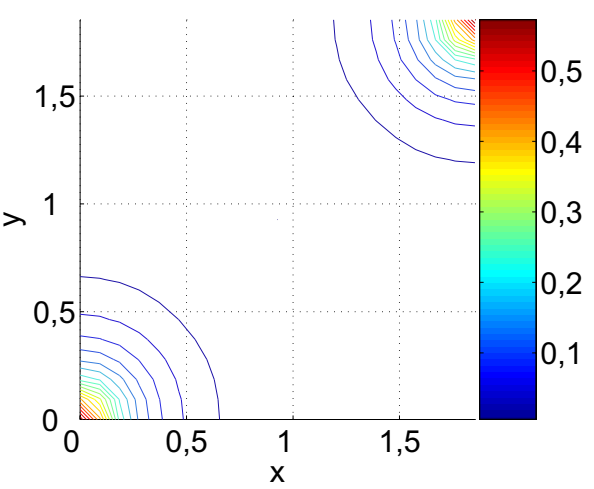

(b)

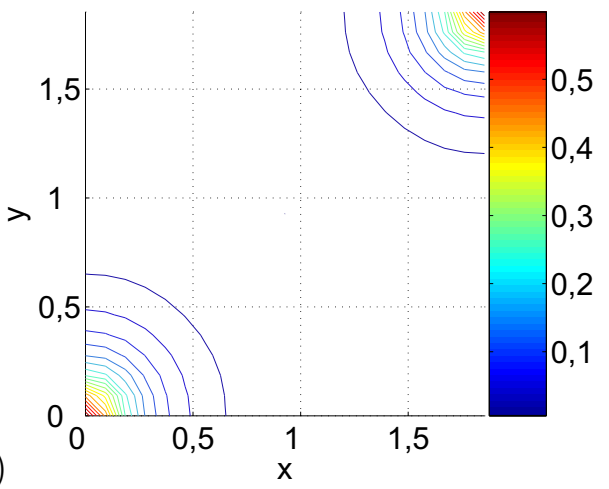

(c)

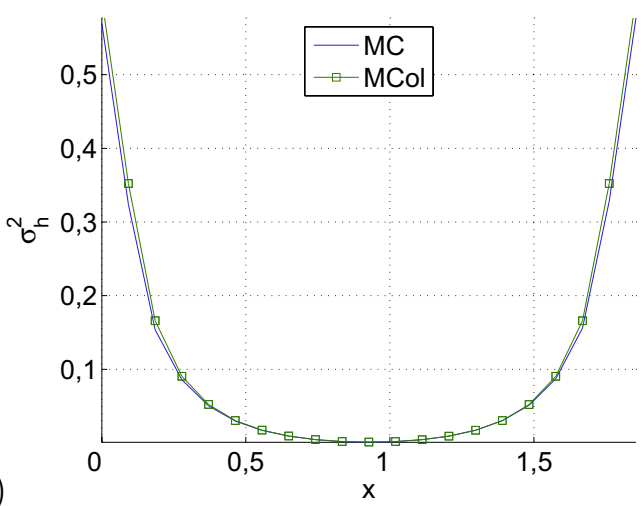

Figura 5 - Gráfico de Contorno da Variância: (a) MC e (b) MCol. A Fig. (c) representa os perfis da diagonal com $\eta=4 \mathrm{e} \sigma_{Y}^{2}=1$.

Nenhum fluxo é prescrito sob a parte inferior ou superior do aqǘfero e 0 fluxo nas fronteiras laterais se estende para 0 infinito com carga hidráulica $H_{0}=380 \mathrm{~m}$ e porosidade efetiva $\psi_{e}=25 \%$. Nos exemplos a seguir, estabelecemos as condições de fronteira por meio da fórmula de Theis (8). Discutimos 0 uso da fórmula de Boulton (6) e 0 arranjo dos poços na próxima seção.

No MCol se considerou o polinômio de caos de grau $P=2$ e a dimensão estocástica $M=6$. Nas Figuras 6,7 e 8 estão apresentados os mapas de iso-contorno da carga hidráulica e os perfis hidráulicos do MC e MCol nos casos ( $\left.\eta=1, \sigma_{Y}^{2}=1\right)$, $\left(\eta=4, \sigma_{Y}^{2}=1\right)$ e $\left(\eta=100, \sigma_{Y}^{2}=1\right)$ respectivamente.

Através dos gráficos de perfil Figuras 6(c), 7(c) e 8(c) podemos notar que não ocorre grande diferença nos resultados da média mesmo sob baixo coeficiente de correlação, por causa das condições de fronteira.

É observado também que o MCol não foi capaz de desenvolver o mesmo resultado da média na variância. Segundo Le Maître et al. (2003) o problema está relacionado à dimensão estocástica muito baixa, visto que as autofunções de alta freqüência no caso dos senos e cossenos com coeficientes elevados (vide Eq. (24)) são excluídas da expansão de Karhunen-Loève e com isso as variações curtas causadas pelo baixo comprimento de correlação não são capturadas e a variância fica subestimada. Entretanto, à medida que a correlação espacial aumenta existe uma melhora significativa na escala da variância (vide Fig. 11). Isto acontece porque quanto maior 0 coeficiente de correlação, mais rapidamente os autovalores em (22) tendem a zero e mais rápida é a convergência da expansão (5), ou seja, o erro de truncamento da expansão (5) diminui com o aumento de $\eta$.

\section{Otimização da exploração do aqüífero freático}

Inicialmente se propõe adotar uma bateria ótima contendo um número fixo de poços. A configuração da futura bateria pode ser ampliada em etapas sucessivas de modo que em cada uma delas sejam satisfeitos os critérios de otimização especificados como a seleção do espaçamento adequado, a geometria e as dimensões da bateria de extração de poços. Na seleção deste espaçamento, considerações técnicas e econômicas estão envolvidas. Quanto mais afastados estiverem os poços menor será a interferência entre eles, porém terá um alto custo com tubulações e instalações elétricas (Hantush, 1964). 
(a)

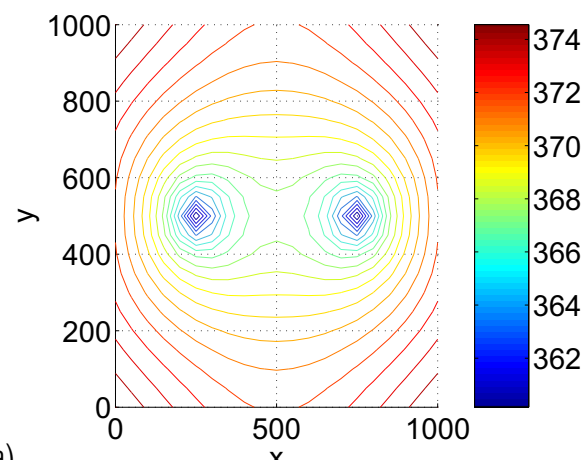

$\mathrm{x}$

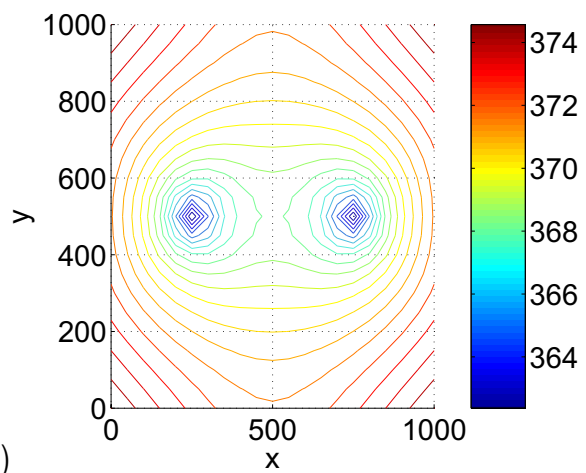

(b)

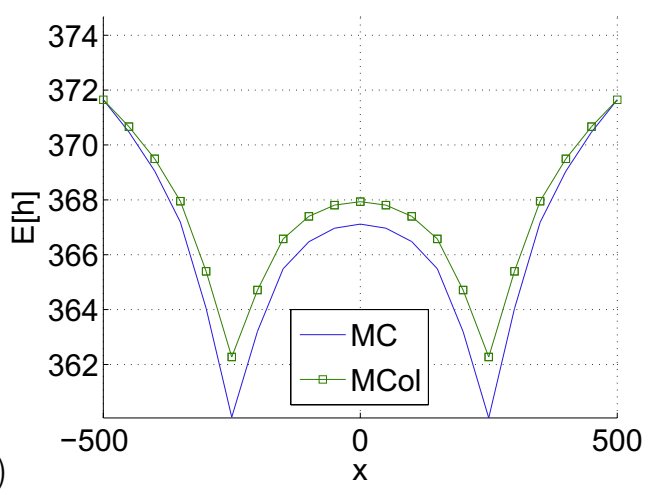

Figura 6 - Média da carga hidráulica gerada pela interferência entre dois poços, em linha separados por uma distância $d_{m}=460 \mathrm{~m}$ com $\eta=1$. (a) MC (b) MCol. A Fig. (c) estabelece os respectivos perfis.

(a)

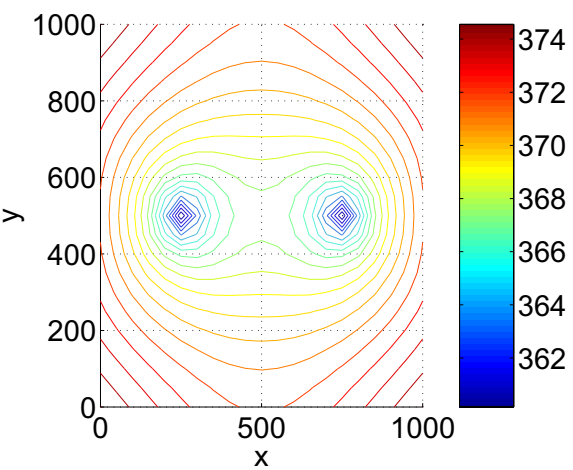

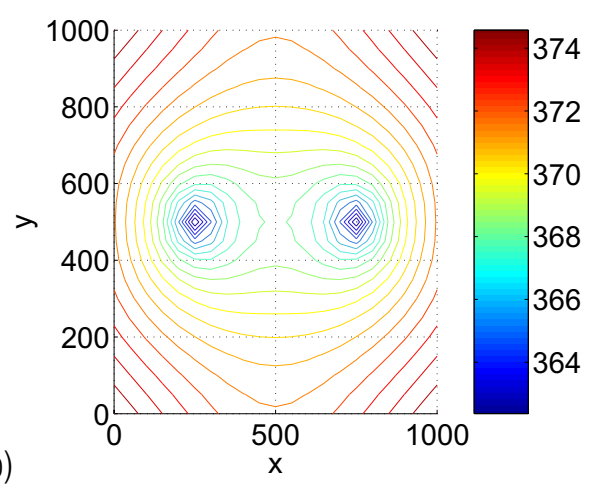

(b)

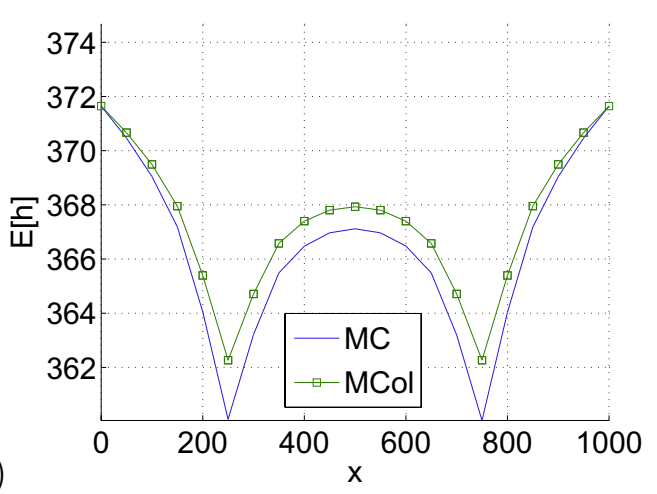

Figura 7 - Média da carga hidráulica gerada pela interferência entre dois poços, em linha separados por uma distância $d_{m}=460 \mathrm{~m}$ $\operatorname{com} \eta=4$. (a) MC (b) MCol. A Fig. (c) estabelece os respectivos perfis. 
(a)

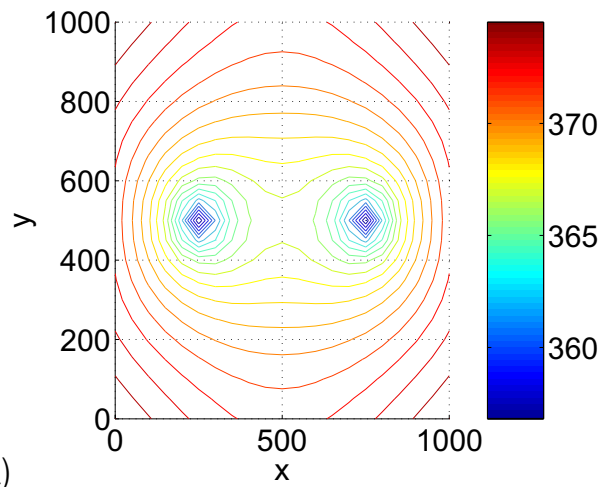

(b)

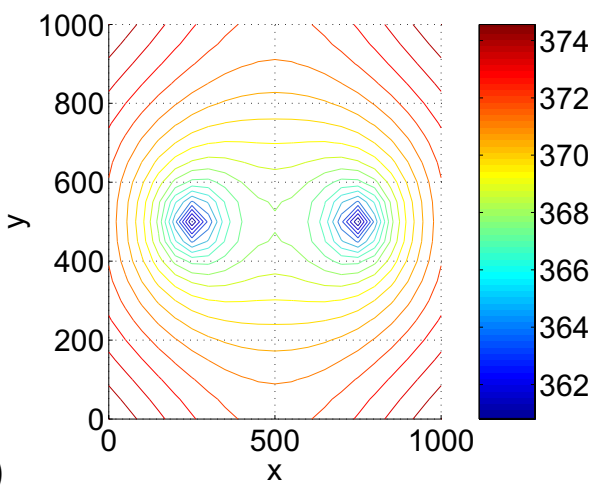

(c)

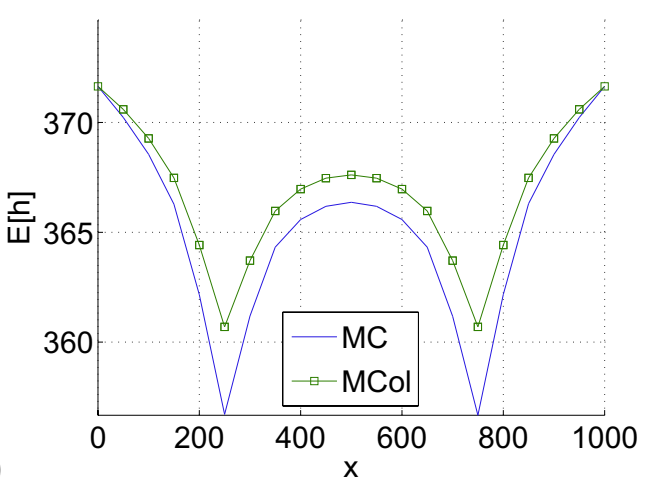

Figura 8 - Média da carga hidráulica gerada pela interferência entre dois poços, em linha separados por uma distância $d_{m}=460 \mathrm{~m}$ com $\eta=100$. (a) MC (b) MCol. A Fig. (c) estabelece os respectivos perfis.

A fim de reduzir este problema, Cavalcanti (2006) propôs um esquema de exploração otimizado do aqüífero Recôncavo na bacia do rio Capivara, usando a fórmula de Theis (8) e supondo meio homogêneo e isotrópico. Complementando o trabalho de Cavalcanti (2006), utilizamos também a fórmula de Boulton como solução analítica no cálculo da carga hidráulica.

Note que a análise das informações coletadas durante 0 teste de bombeamento depende largamente da seleção da equação fluxo-poço aplicável no sistema de investigação e da concordância com que as suposições básicas governam estas equações e as características do aqüífero estudado.

Seguindo Cavalcanti (2006), consideramos adequado um rebaixamento máximo da ordem de $13 \%$ de $H_{0}$, a espessura saturada média do aqǘfero, ou seja, $s_{\max }=50 \mathrm{~m}$. Definido como critério de otimização o valor de rebaixamento máximo permissível $s_{\max }$, é possível determinar a distância mínima $d_{m}$ entre poços de produção numa bateria genérica de extração. No presente trabalho o rebaixamento máximo permissível é definido como o rebaixamento criado por um único poço acrescido de $10 \%$ desse valor a ser causado pela interferência de cada poço da bateria, isto é, se $s_{w}=0,13 H_{0}$ é 0 rebaixamento de um dos poços numa bateria de dois poços, o rebaixamento máximo permissível na bateria é

$$
s_{\max }=s_{w}+0,1 s_{w} .
$$

No caso de três poços, o rebaixamento $s_{\max }$ é expresso por

$$
s_{\max }=s_{w}+0,1 s_{w}+0,01 s_{w},
$$

e de forma análoga para os demais poços a serem inseridos.

Adotando-se os valores característicos para geometria e propriedades hidráulicas do aqüífero Recôncavo e 0 critério de otimização referido anteriormente calculamos as distâncias mínimas entre os poços para baterias com dois e três poços através das Eqs. (11) e (12) respectivamente, supondo o tempo de exploração contínua de um ano de duração. Os mapas de contorno das distribuição do potencial foram calculados pelo método de elementos finitos com funções de base contínuas e bilineares por partes cuja malha considerada foi $40 \times 40$ elementos quadrados.

As Figuras 12(a) e 12 (b) foram calculadas através das fórmulas de Theis e de Boulton respectivamente, e mostram 0 es- 
(a)

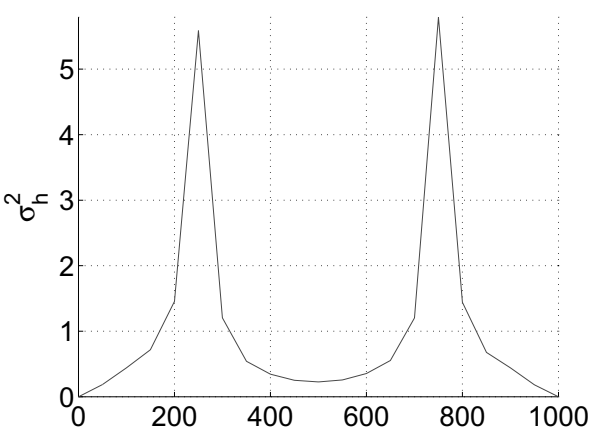

(b)

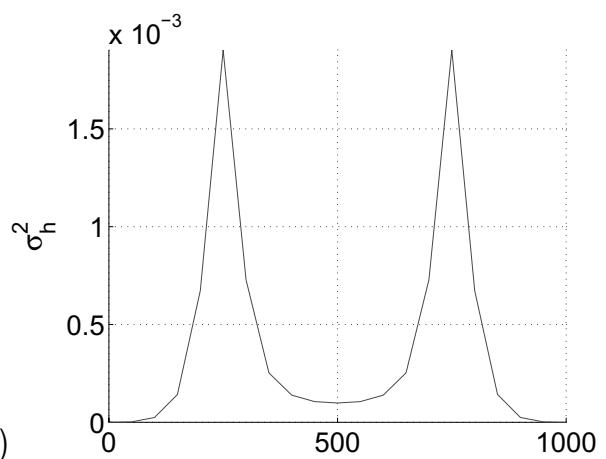

Figura 9 - Perfil da Variância da carga hidráulica gerada pela interferência entre dois poços quando $\eta=1$. (a) MC (b) MCol.

(a)

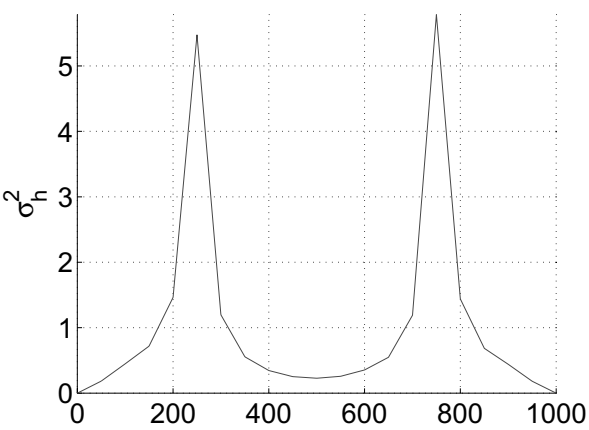

(b)

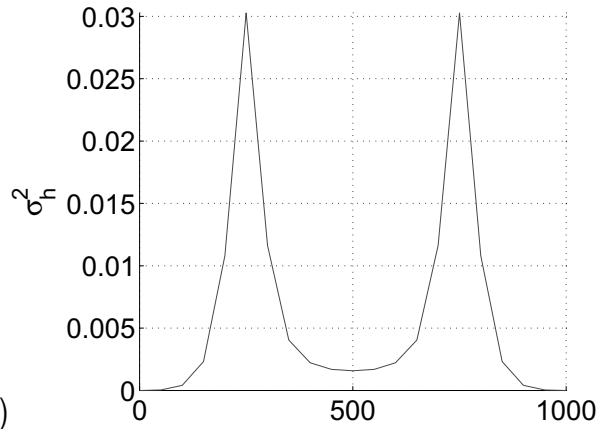

Figura 10 - Perfil da Variância da carga hidráulica gerada pela interferência entre dois poços quando $\eta=4$. (a) MC (b) MCol.

(a)

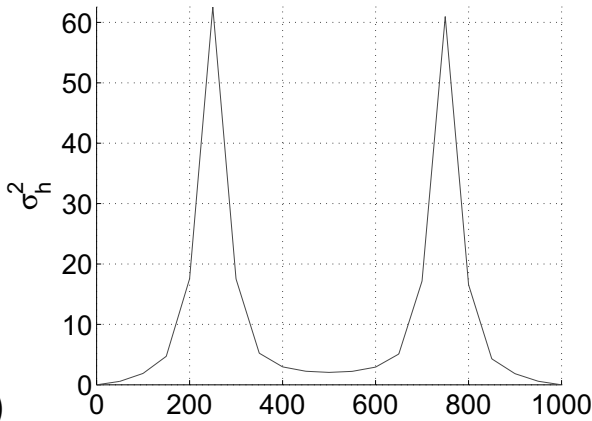

(b)

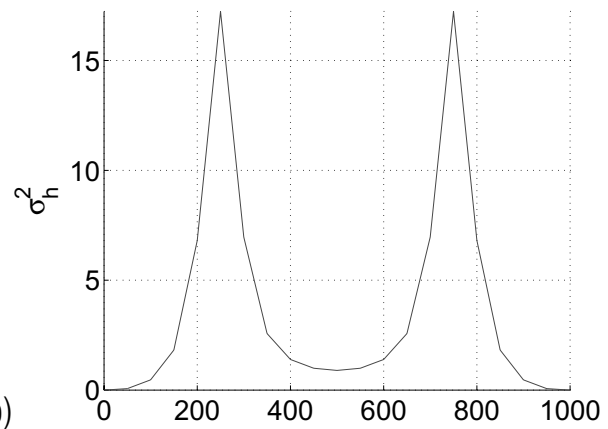

Figura 11 - Perfil da Variância da carga hidráulica gerada pela interferência entre dois poços quando $\eta=100$. (a) MC (b) MCol.

quema de dois poços freáticos com descargas uniformes. A Figura 12(c) mostra o perfil hidráulico entre os poços utilizando ambas as fórmulas de Boulton e Theis.

No caso de três poços usamos a distância calculada por (Cavalcanti, 2006) e concluímos que a geometria é a de um triângulo equilátero de lado $d_{m}=700 \mathrm{~m}$ (vide Fig. 13).

Assim é possível com base na metodologia proposta fazer um planejamento eficiente e permanente da exploração de um aqüífero sob o princípio de superposição. Inicialmente propomos adotar uma bateria ótima contendo um número fixado de poços. A configuração da futura bateria pode ser ampliada, em etapas sucessivas, de modo que em cada uma delas sejam satisfeitos os critérios de otimização especificados. Após planejar a geometria e as dimensões de uma bateria de extração de poços, critérios de natureza econômica devem ser introduzidos visando otimizar a preservação e a distribuição de água explorada. 
(a)

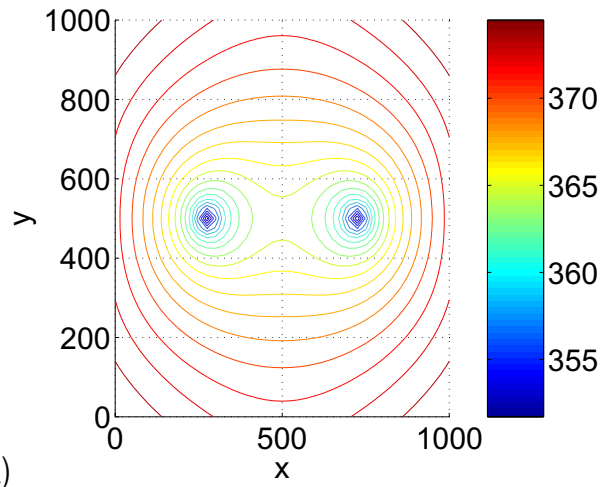

(b)

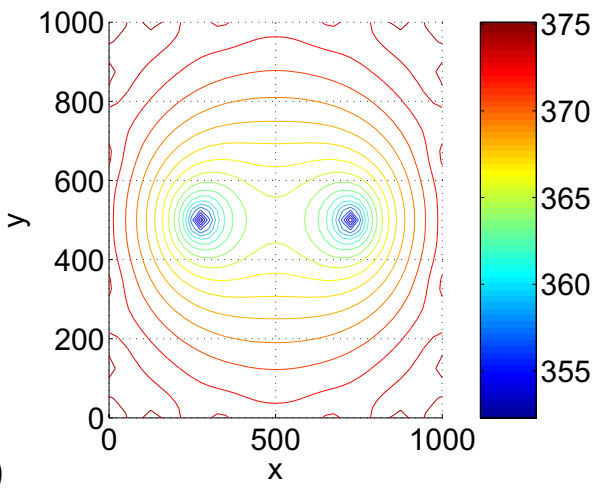

(c)

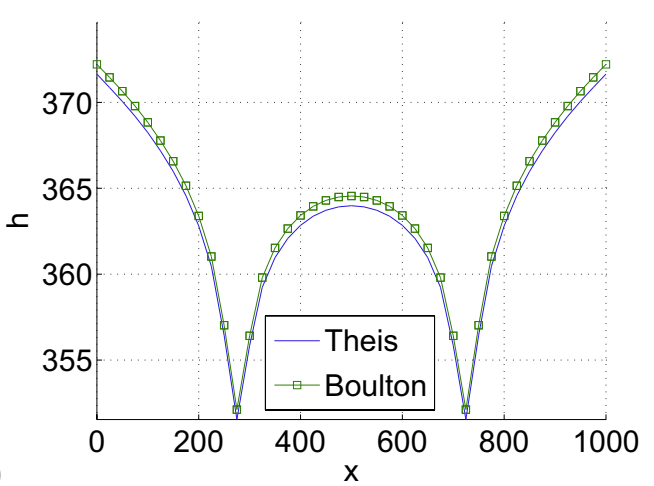

Figura 12 - Carga hidráulica gerada pela interferência entre dois poços, em linha separados por uma distância $d_{m}=460 \mathrm{~m}$. (a) fórmula de Theis, (b) fórmula de Boulton, (c) estabelece os respectivos perfis.
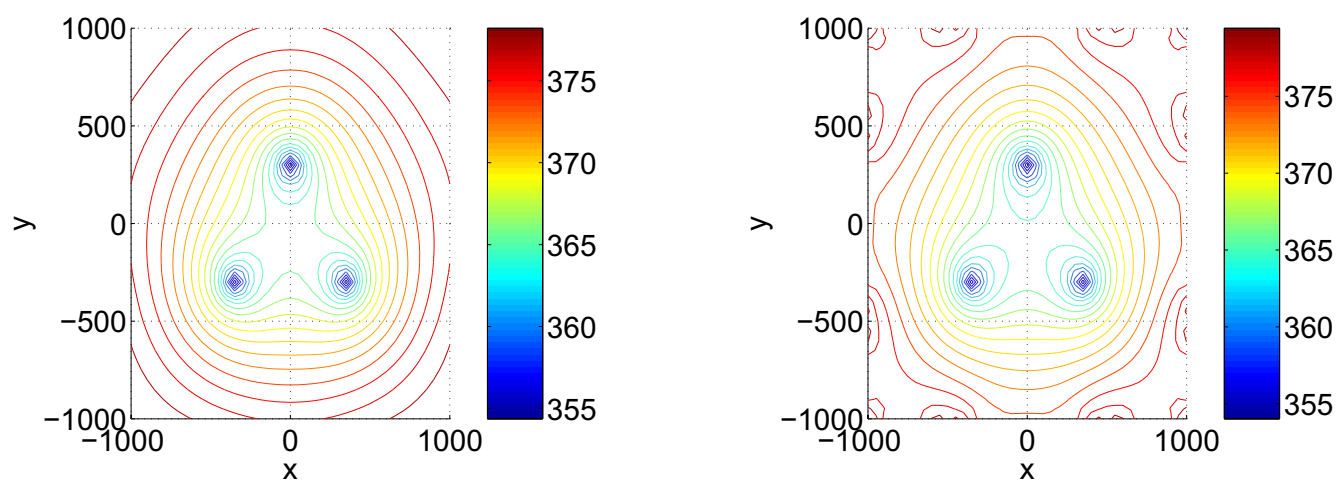

Figura 13 - Carga hidráulica gerada pela interferência entre três poços, formando um triângulo eqüilátero de lado $d_{m}=700 \mathrm{~m}$. Direita: fórmula de Theis; Esquerda: fórmula de Boulton.

A análise das informações coletadas durante testes de bombeamento depende largamente da seleção da equação fluxo-poço aplicável no sistema de investigação e da concordância com as suposições básicas que governam estas equações e as características do aqüífero estudado.

As variações de rebaixamento com o tempo e distâncias dos poços de bombeamento podem ser interpretadas em vários caminhos se outras informações não são avaliadas trazendo, por conta disto, resultados imprecisos. Todavia, se os dados são cuidadosamente estudados e todas as possíveis interpretações são consideradas, as análises destes parâmetros podem prover informações valiosas não somente sobre as propriedades hidráulicas, mas também sobre as condições físicas e os parâmetros geométricos do sistema de fluxo tais como: a natureza e localização das fronteiras hidráulicas, a espessura média do aqüífero, 0 raio do poço e as perdas hidráulicas. 


\section{CONCLUSÕES}

Modelos estocásticos para fluxo transiente em zonas saturadas são desenvolvidos neste estudo. Estabelecidas as características hidrogeológicas, soluções analíticas aproximadas foram usadas para hidráulica transiente de poços em aqǘferos livres e extensos em meios homogêneos e isotrópicos. Depois se ampliou a metodologia para meios heterogêneos com a inclusão de parâmetros aleatórios.

Observamos no caso estacionário que a baixa correlação entre as variáveis espaciais afeta a solução média da carga hidráulica atribuído ao MCol. Porém, o aumento na correlação das variáveis espaciais traz resultados compatíveis com o MC.

Em regime transiente, as condições de fronteira mantiveram os resultados da média estáveis mesmo sob um baixo coeficiente de correlação. Isto porém não se refletiu na variância que, em virtude do baixo valor da dimensão estocástica $M$, acumulou erros nas simulações.

\section{AGRADECIMENTOS}

Agradecemos os comentários de Marcus Sarkis e Juan Galvis (IMPA). Os autores contaram com 0 apoio da CAPES, CPGGUFBA e do programa PROCAD/CAPES (IMPA-UFPR).

\section{REFERÊNCIAS}

BABUŠKA I, NOBILE F \& TEMPONE R. 2007. A stochastic collocation method for elliptic partial differential equations with random input data. SIAM J. Numer. Anal., 45(3): 1005-1034.

CAVALCANTI SS. 2006. Quantificação dos recursos hídricos subterrâneos do aqǘfero Recôncavo na bacia do rio Capivara. Tese de Dou- torado, Universidade Federal da Bahia. 121 p.

CHAKRABORTY S \& DEY S. 1995. Stochastic finite element method for spatial distribution of material properties and external loading. Comput. Struct., 41: 41-45.

CORREA M \& LOULA A. 2008. Unconditionally stable mixed finite element methods for Darcy flow. Comput. Methods Appl. Mech. Engrg., 197: 1525-1540.

EVANS L. 1998. Partial Differential Equations. Grad. Stud. Math., 19, AMS, Providence, RI. 662 pp.

HANTUSH M. 1964. Hydraulics of wells. In: CHOW VT (Ed.). Advances in Hydrosciences. vol. 1, Academic Press, New York, 281-432.

HUGHES TJR. 1987. The finite element method. Linear static and dynamic finite element analysis. Prentice Hall Inc., Englewood Cliffs, NJ, $803 \mathrm{pp}$.

HUNT B \& SCOTT D. 2005. Extension of Hantush and Boulton solutions. J. Hydrol. Eng., 10(3): 223-236.

LE MAÎTRE 0, KNIO 0, DEBUSSCHERE B, NAJM H \& GHANEM R. 2003. A multigrid solver for two-dimensional stochastic diffusion equations. Comput. Methods Appl. Mech. Engrg., 192: 4723-4744.

RUPERT C \& MILLER C. 2007. An analysis of polynomial chaos approximations for modeling single-fluid-phase flow in porous medium systems. J. Comput. Phys., 226: 2175-2205.

ZHANG D \& LU Z. 2002. Stochastic analysis of flow in heterogeneous unsaturated-saturated system. Water Resour. Res., 38(2): 10.1-10.15.

ZHANG D \& LU Z. 2004. An efficient, high-order perturbation approach for flow in random porous media via Karhunen-Loève and polynomial expansions. J. Comput. Phys., 194: 773-794.

\section{NOTAS SOBRE OS AUTORES}

Juarez dos Santos Azevedo é graduado em Matemática pela Universidade Estadual de Feira de Santana, Feira de Santana, BA, em 2001. Mestrado em Matemática, Universidade Federal da Bahia, Salvador, BA, em 2003. Doutorado no Programa de Pós-graduação em Geofísica da Universidade Federal da Bahia em 2009. Atualmente é Bolsista de Desenvolvimento Tecnológico Industrial do CNPq no Laboratório Nacional de Computação Científica. Tem interesse na análise de métodos estocásticos no estudo de escoamento de fluido em meios porosos.

Saulo Pomponet Oliveira é graduado em Matemática Aplicada e Computacional pela Universidade Estadual de Campinas, Campinas, SP, em 1997. Mestrado em Matemática Aplicada, Universidade Estadual de Campinas, Campinas, SP, em 1998. Doutorado em Matemática Aplicada, University of Colorado, Denver, Estados Unidos, em 2003. Foi pesquisador visitante no CPGG-UFBA e no Osservatorio Geofisico Sperimentale, Trieste, Itália. Atualmente é professor do Departamento de Matemática da Universidade Federal do Paraná. Tem interesse na análise de problemas de propagação de ondas com métodos de elementos finitos.

Olivar Antônio Lima de Lima é graduado em Geologia (1967) e Mestre em Geologia Estrutural (1970) pela Universidade Federal do Rio de Janeiro (UFRJ). Doutor em Geofísica (1979) pela Universidade Federal da Bahia (UFBA). Pós-Doutorado (1989-1990) no Departamento de Engenharia de Petróleo da Universidade do Texas, Austin. Professor Titular da UFBA. Áreas de atuação: Aplicação de métodos geofísicos na exploração de água subterrânea, petróleo e problemas ambientais; Petrofísica. É sócio da SBGf, SBG e ABAS; e da SEG, EAGE, AGU e SPWLA. 